\title{
Revealing body proportions of the enigmatic choristodere reptile Khurendukhosaurus from Mongolia
}

Ryoko Matsumoto, Khishigjav Tsogtbaatar, Shinobu Ishigaki, Chinzorig Tsogtbaatar, Zorig Enkhtaivan, and Susan E. Evans

Acta Palaeontologica Polonica 64 (2), 2019: 363-377 doi:https://doi.org/10.4202/app.00561.2018

Khurendukhosaurus is an enigmatic genus of choristodere, recorded from the Lower Cretaceous of East Asia, Mongolia, and Siberian Russia. Until now, it was known only from isolated skull and postcranial elements, limiting comparison with other genera. Three major morphotypes have been recognised within Choristodera: longirostrine neochoristoderes with short-necks, and brevirostrine non-neochoristoderes with either short or long necks. The morphotype of Khurendukhosaurus was uncertain, although it had been inferred to be long-necked, based on cervical and caudal vertebral morphology shared with the Chinese Hyphalosaurus and on the results of phylogenetic analysis that placed it within a clade of Sino-Japanese long-necked taxa. Newly discovered material from the Mongolian type locality, Khuren-Dukh, preserves most major postcranial elements of a single individual. This specimen confirms that Khurendukhosaurus belongs to the long-necked morphotype, in having at least 13 cervical vertebrae. Moreover, a new phylogenetic analysis supports the placement of Khurendukhosaurus as a sister group of the Hyphalosaurus + Shokawa clade. Based on the new material, Khurendukhosaurus is estimated to have been roughly $1 \mathrm{~m}$ in total length, placing it at the upper end of the size range for long-necked choristoderes.

Key words: Reptilia, Choristodera, Khurendukhosaurus, Cretaceous, Mongolia, Khuren-Dukh.

Ryoko Matsumoto [r-matsumoto@nh.kanagawa-museum.jp], Kanagawa Prefectural Museum Natural History, 499 Iryuda, Odawara, Kanagawa, 250-0031, Japan.

Khishigjav Tsogtbaatar [tsogtbaatar@ipg.mn], Chinzorig Tsogtbaatar [chinzorig.tsogtbaatar@gmail.com], Zorig Enkhtaivan [zorig.enkhtaivan99@gmail.com

], Institute of Paleontology and Geology, Mongolian Academy of Sciences,

S. Danzan Street 3/1, Ulaanbaatar-15160, Mongolia. Shinobu Ishigaki [isgk-7591@wind.email.ne.jp ], Okayama University of Science, 1-1 Ridai-cho, Kita-ku, Okayama, 700-0005, Japan. Susan E. Evans [ucgasue@ ucl.ac.uk], Department of Cell and Developmental Biology, UCL, University College London, Gower Street, London WC1E 6BT, UK. 
This is an open-access article distributed under the terms of the Creative Commons Attribution License (for details please see creativecommons.org), which permits unrestricted use, distribution, and reproduction in any medium, provided the original author and source are credited.

FoF Full text $(1,111.1 \mathrm{kB})$

FoF Supplementary file $(71.7 \mathrm{kB})$ 